Contribution à l'étude

\title{
des Microphallidae Travassos, 1920 (Trematoda) des côtes de France
}

\section{Description de deux espèces nouvelles à cycle évolutif abrégé originaires de Corse.}

\author{
Par S. DEBLOCK et P. TRAN VAN KY
}

\section{PLAN}

1. - INTRODUCTION.

2. - Historique des espèces de Microphallidés à cycle évolutif abrégé. Liste, CLASSIFICATION, BIOLOGIE.

3. - DEscription de deux nouveaux cycles de ce type :

a) Maritrema syntomocyclus n. sp. Discussion.

b) Microphallus scolectroma $\mathrm{n}$. sp. Discussion.

4. - Conclusions.

5. - RÉSUMÉ.

\section{1. - INTRODUCTION}

L'autopsie de mollusques Hydrobiidés d'un petit gîte permanent d'eau saumâtre de la côte du Sud de la Corse a révélé en été 1965 la présence de métacercaires de deux trématodes différents appartenant à la famille des Microphallidés. Nous devons nos remerciements les plus chaleureux et les plus sincères à nos amis les Professeurs J.-M. Doby et A.-G. Chabaud, pour les facilités de déplacement et de travail qu'ils ont si aimablement mises à notre disposition, sans lesquelles nous n'aurions pu parfaire l'étude de ces parasites. Les cycles larvaires que tous deux accomplissent en totalité chez le mollusque hôte intermédiaire les signalent à l'attention; c'est en effet une modalité évolutive restreinte à de rares espèces de la famille des Microphallidés, toutes 
les autres passant obligatoirement par un hôte intermédiaire supplémentaire, constitué habituellement par un crustacé.

\section{2. - HISTORIQUE}

Sur un peu plus de 100 espèces réparties en une vingtaine de genres qui composent la famille des Microphallidae, celles à cycles ne comportant que 2 hôtes sont actuellement au nombre de 5 , distribuées dans 4 genres différents. Les voici, par ordre chronologique de découverte, suivies de l'inventeur du cycle:

$1^{\circ}$ Maritrema oocysta (Lebour, 1907), par Rothschild, 1937 (1) ;

$2^{\circ}$ Microphallus pygmoeun (Levinsen, 1881), par Belopolskaïa, 1949 ;

$3^{\circ}$ Microphallus somateriae (Kulatschkowa, 1953), par Tchoubryck, 1957 et Kulatschkowa, 1958 (in Belopolskaïa, 1962) ;

$4^{\circ}$ Atriophallophorus minutus (Price, 1934), par Stunkard, 1958 ;

$5^{\circ}$ Levinseniella sp. Rebecq, 1964, par l'auteur $\left(1^{\text {bis }}\right)$.

A côté de ces descriptions fondamentales, la présence de métacercaires de Trématodes Microphallidés enkystées chez des mollusques a été signalée plusieurs fois en Europe et notamment en mer Baltique par Sinitzin, 1911 (in Rothschild, 1937, et Belopolsk., 1963), par Reimer, 1963 (espèce rapportée à Microphallus claviformis), et peut-être aussi par Markowski en 1936. Enfin, en Grande-Bretagne, Lebour (1907) décrivait (Meta)-cercaria pirum en même temps que (Meta)-cercaria oocysta.

Le tableau I résume les principales connaissances morphologiques que nous avons de ces larves de Trématodes classées selon Belopolskaïa (1962) en fonction des modalités biologiques des cycles.

$1^{\circ}$ Le premier groupe comporte les Microphallidés à cycle classique à trois hôtes : deux intermédiaires et un définitif, selon le schéma Mollusque-Crustacé-Vertébré. Nous avons exposé dans des articles antérieurs la somme des connaissances biologiques concernant deux genres parmi les mieux représentés: le genre Maritrema (in Deblock et coll., 1961) et le genre Microphallus (in Deblock et coll., 1965). Sur la trentaine d'espèces dont chacun se constitue, cinq Maritrema et quatre Microphallus évoluent de cette façon (2), sans compter leurs très nombreux représentants dont le second hôte crustacé est connu.

(1) L'auteur s'est borné à rapporter le fait incidemment sans fournir ni précision sur ses modalités, ni iconographie.

$\left(1^{\text {bis }}\right)$ Voir addendum.

(2) Il est permis d'émettre en effet quelques réserves quant à l'exactitude de certains cycles et, par voie de conséquence, à la nature du premier hôte intermédiaire et celle de la cercaire qu'il émet. Les recherches les plus récentes semblent préciser que toutes les véritables cercaires de Microphallide sont des Xiphidiocercaires monostomes agastres leptocerques (cas de Maritrema subdolum et laricola; Mecynophallus uca; Microphallus nicolli, similis, papillorobustus, claviformis; Megallophallus diodontis; Levinseniella amnicolae; Levinseniella sp. Rebecq). Etant donné la grande homogénéité de la famille et des genres qui la composent, il paraît de plus en plus suspect que certaines espèces puissent présenter des formes larvaires aussi différentes des précédentes que des Xiphidiocercaires distomes pourvues de tube digestif et de pharynx qu'ont décrites les auteurs dans les cas de Mar. caridine, Mar. obstipum et Mic. pirum; on sait enfin que le cycle de $M$. rhodanicum (syn. de $M$. subdolum, Rebecq, 1960) est faux (Deblock et coll., 1961; Rebecq, 1964). 
$2^{\circ}$ Le second groupe comporte tous les Microphallidés à cycle abrégé, ne comportant plus que deux hôtes, le mollusque se substituant au crustacé en tant que second hôte intermédiaire. Cette simplification évolutive s'accompagne souvent de particularités qui conduisent à deux sous-groupes.

A) Le premier sous-groupe comporte les espèces où la suppression du stade crustacé $2^{e}$ hôte intermédiaire n'a pas entraîné d'autres modifications; les cercaires notamment sont classiques. Il s'agit des espèces Mar. oocysta, Levinseniella sp. et Microphallus somateriae.

B) Le second sous-groupe comporte les espèces où la corollaire du raccourcissement du cycle est la modification de la cercaire dans le sens d'une adaptation évolutive monstrueuse : l'absence de stade aquatique libre entraîne la perte de l'appendice caudal propulsif, et l'absence de second hôte intermédiaire, celle du stylet. C'est le cas d'Atriophallophorus minutus et de Microphallus pygmoeum. Cette dernière espèce se singularise encore davantage par le non-enkystement de la métacercaire, qui accomplit tout son développement à l'état libre dans le sporocyste; elle ne forme jamais de kyste (3).

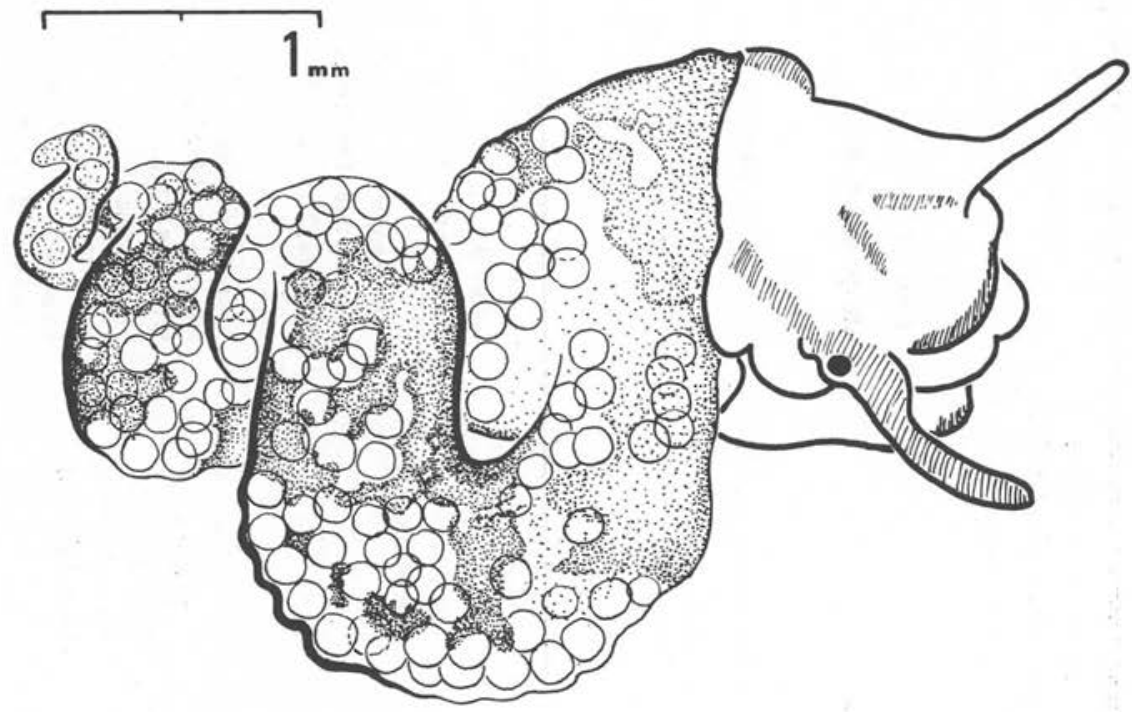

FIG. 1. - Maritrema syntomocyclus n. sp. - Aspect in vivo du parasitisme d'un exemplaire décoquillé d'Hydrobia acuta. Seuls les kystes situés immédiatement sous la membrane non pigmentée du manteau sont visibles. L'aspect du parasitisme par Microphallus scolectroma est identique.

(3) Certaines modalités de l'évolution chez le mollusque n'ont pas toujours été précisées. C'est ainsi qu'on ignore si la cercaire de chacune des espèces évolue en métacercaire à l'intérieur même du sporocyste secondaire ou si elle en émerge pour achever son évolution au sein des tissus de l'hôte. Le premier mode est le fait de $M$. pygmoeum; chez Metacercaria pirum Lebour et Levinseniella $\mathrm{sp}$. Rebecq, la libération du kyste de la métacercaire mûre est un phénomène secondaire à la destruction spontanée du sporocyste vieilli; il se retrouve donc dans l'hépato-pancréas à la suite d'un processus passif; le second mode, actif, serait le fait de la cercaire d'Atriophallophorus minutus selon Stunkard. On ne connaît le mode ni pour Mar. oocysta ni pour Mic. somateriae. 


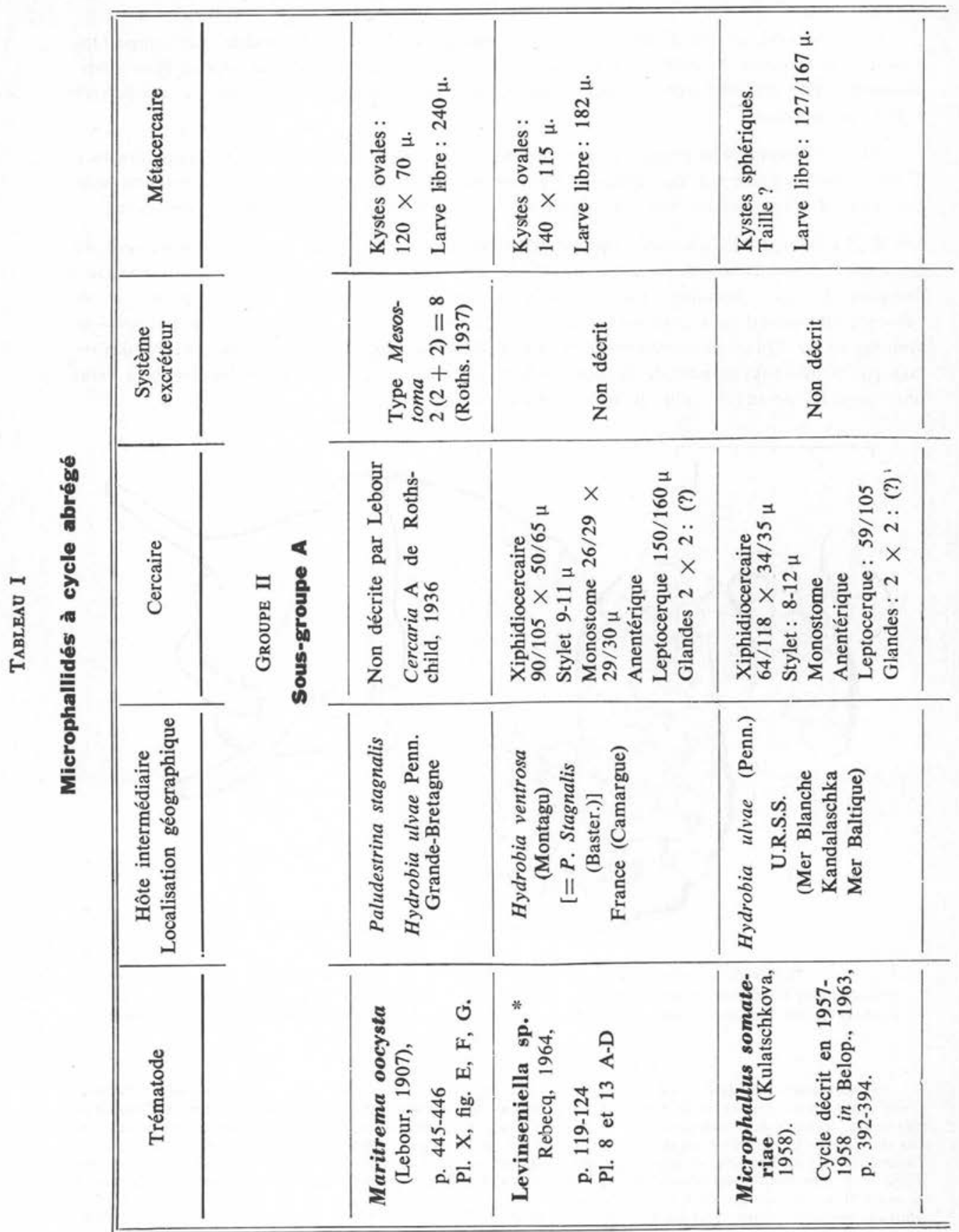




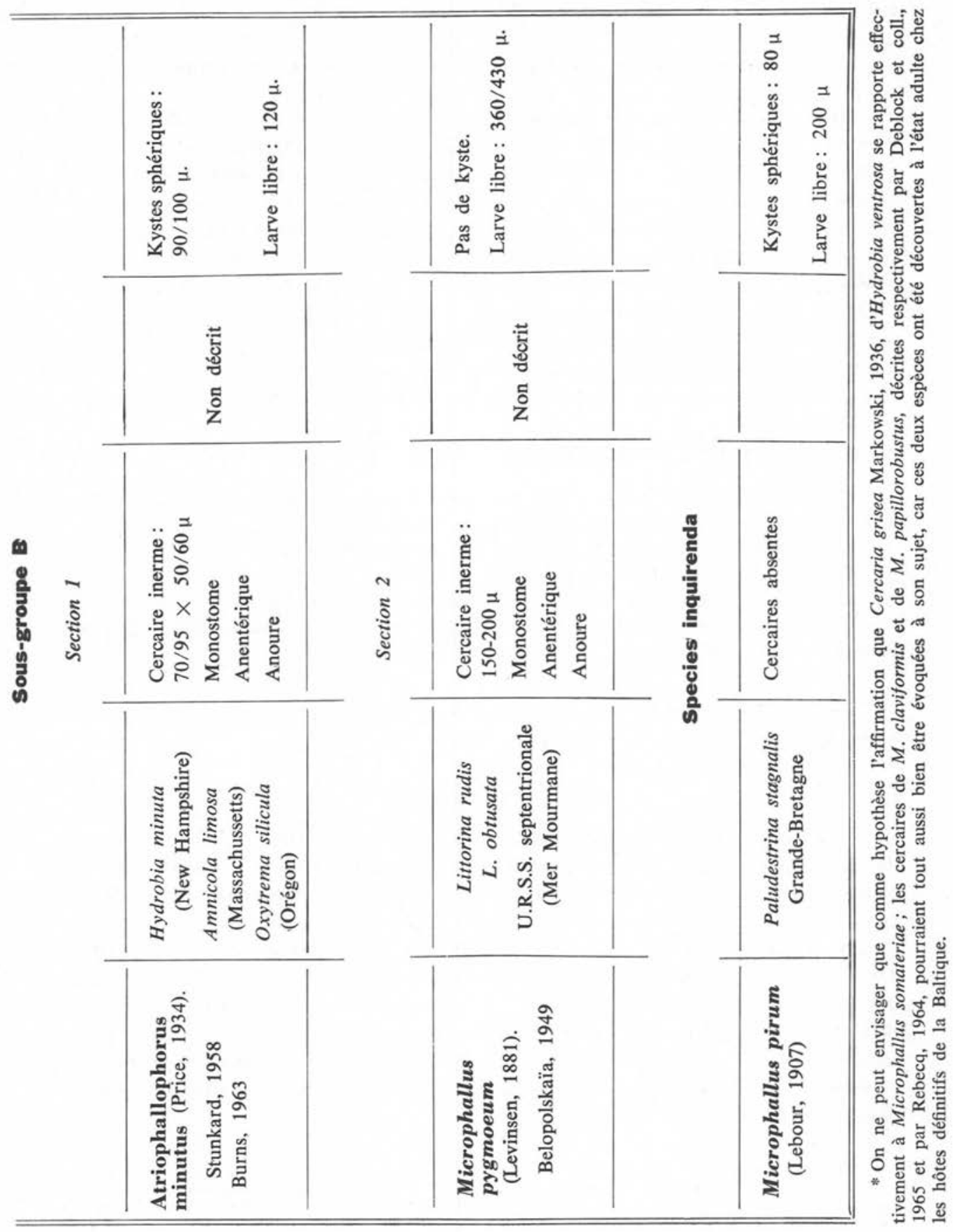




\section{3. - DESCRIPTION DE DEUX NOUVEAUX CYCLES ABREGES}

Sur un total de 452 mollusques, 56 sont parasités, soit $12 \% .8$ présentent des cercaires évoquant celles de Microphallus claviformis sensu Deblock et coll., 1965 ; 1 des cercaires évoquant celles de Maritrema subdolum sensu Deblock et coll., 1961 ; 17 une grosse cercaire ocellée non déterminée; et 2 des formes larvaires immatures. 28 , soit $6 \%$, contenaient des kystes de métacercaires de Microphallidés, dans la proportion approximative, qui ne fut précisée que secondairement, d'un tiers d'un Microphallus pour deux tiers d'un Maritrema. Dans les limites de notre observation, il n'y a pas d'infestation mixte. Lors de la dissection d'un mollusque atteint d'un tel parasitisme, les métacercaires enkystées s'échappent facilement des tissus de l'hôte dilacéré. L'écrasement ménagé de l'hépato-pancréas entre lame et lamelle ou une plus fine dissection libèrent quelques sporocystes intacts ou fragmentés, ou leur contenu (4).

\section{A) MARITREMA SYNTOMOCYCLUS n. sp.}

HôTE DÉFINITIF : inconnu. Il s'agit vraisemblablement d'oiseaux malacophages (Charadriiformes ou Ansériformes) habitués des rivages maritimes.

Localisation géographiQue: Golfe de Figari (Corse, France).

DATE DE RÉCOLTE : juillet 1965.

MATÉRIEL DE DESCRIPTION : matériel vivant et fixé. Coupes histologiques.

Hôte INTERmÉdiaire: Hydrobia (= Paludestrina) acuta (Draparnaud), Mollusque Prosobranche Bythinellidé.

Nous décrirons les formes larvaires dans l'ordre suivant: sporocystes, cercaires, kyste de la métacercaire et métacercaire dékystée.

\section{$1^{\circ}$ Sporocystes}

Les nombreux sporocystes secondaires ne parasitent aucune région préférentielle de l'hépatopancréas du mollusque ou de la glande génitale (fig. 1). Ce sont des sacs cylindriques non diverticulés, incolores, à parois minces, relativement peu adhérents aux tissus qui les hébergent. Leur taille dépend du nombre de métacercaires mûres qui s'y sont développées. C'est ainsi qu'un sporocyste à quatre métacercaires mûres mesure $450 \times 125 \mu$. Pratiquement immobiles immédiatement après leur libération mécanique des tissus de l'hôte, ils contiennent: $1^{\circ}$ d'une à sept métacercaires mûres ou en voie de maturation, alignées en file le plus souvent, mais parfois côte à côte ; $2^{\circ}$ un nombre toujours restreint de cercaires mûres - de zéro à une, deux ou trois au maximum et mobiles; $3^{\circ}$ des bourgeons pariétaux immobiles sensiblement sphériques composés d'un nombre variable de cellules (fig. 2).

(4) La paroi externe de plusieurs coquilles de mollusques, parasités ou non, porte d'un à quatre ou cinq kystes hémisphériques de 120 à $160 \mu$ de diamètre, à parois claires et à contenu brunâtre et qui lui sont intimement accolés. Ils représentent une métacercaire crevée de Trématode qu'il ne nous a pas été possible d'identifier; il est néanmoins possible qu'il s'agisse de l'une ou l'autre des espèces décrites ci-après. 

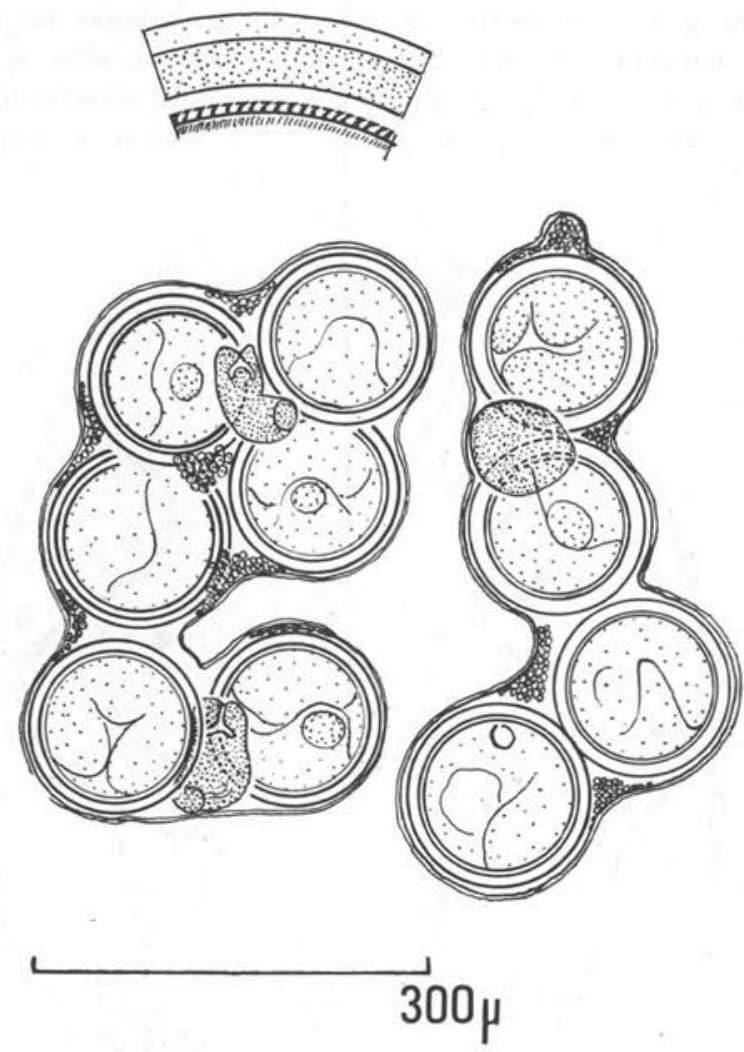

Fig. 2. - Maritrema syntomocyclus n. sp. - Deux sporocystes isolés contenant des bourgeons cercariens, des cercaires et des métacercaires mûres. Au-dessus, fragment de la paroi du kyste et cuticule spinulée.

\section{$2^{\circ}$ Cercaires}

Le raccourcissement du cycle évolutif s'accompagne d'une simplification morphologique de la cercaire: elle perd queue et stylet. L'examen direct à frais ne laisse voir qu'une anatomie restreinte à une ventouse orale antérieure et à une vessie excrétrice postérieure. Distinguer les autres détails nécessite un examen prolongé extrêmement attentif ; les tissus néanmoins donnent une impression générale de parfaitement achevé, et de maturité complète (fig. 3).

La taille varie suivant l'activité de la larve. Immobile, elle mesure $70 \times 50 \mu$; étirée par reptation, elle atteint $80 / 90 \times 40 / 45 \mu$. La cuticule est mince $(0,5$ à $0,75 \mu)$, très finement épineuse et dépourvue de toute soie cuticulaire sensitive. La ventouse orale, sub-termino-ventrale, mesure $15 / 22 \mu$ de large $\times 15 \mu$ de hauteur; elle est 
toujours dépourvue de stylet. Il existe des cellules de pénétration très mal visibles car peu différenciées; au nombre de deux paires droite et gauche, elles se situent latéralement, à peu près à la file les unes des autres, dans la moitié postérieure du corps, sans dépasser le niveau antérieur de la vésicule excrétrice. Peu développées et pourvues

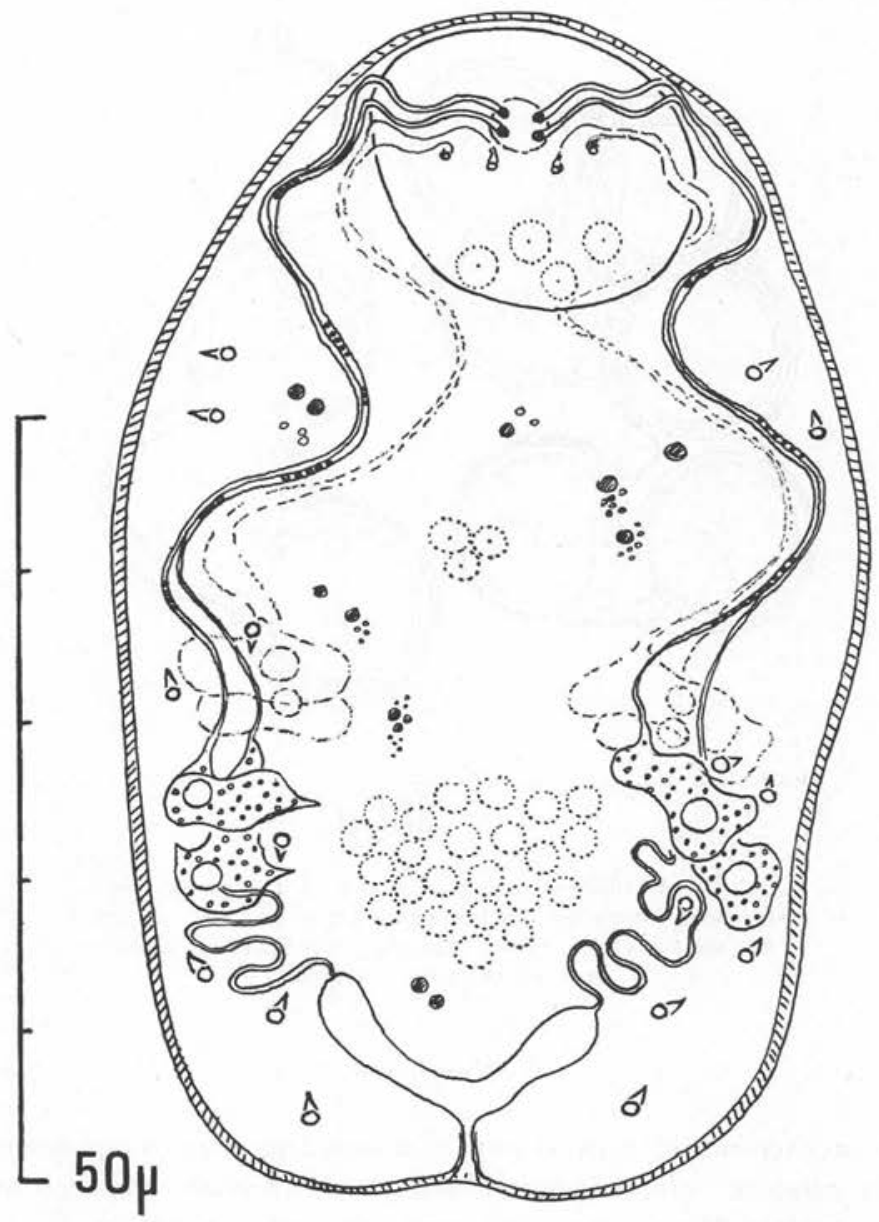

FIg. 3. - Maritrema syntomocyclus n. sp. - Cercaire mûre. Vue ventrale. Ventouse orale en vue apicale.

chacune d'un gros noyau, les deux plus antérieures sont plus ou moins superposées et finement granuleuses, tandis que les deux plus postérieures, disposées en tandem, contiennent des granulations plus volumineuses. Toutes se prolongent antérieurement d'un long canal excréteur grêle et filiforme peu visible, qui décrit une triple sinuosité un peu à la manière de ceux de Cercaria Maritrema subdolum (Deblock et coll., 1961 ; 
Rebecq, 1964). Les quatre canaux cheminent primitivement de concert dans le premier tiers de leur parcours; puis ils se séparent deux à deux pour contourner dorsalement la ventouse orale. Deux fois deux pores excréteurs s'ouvrent à l'apex de la ventouse, au point qui correspondrait à la pointe du stylet, s'il existait ; ils représentent la terminaison cuticulaire des deux canaux les plus externes, très jointifs. Plus ventralement se situent deux autres pores, plus largement séparés, qui correspondent aux canaux les plus internes.

Le système excréteur est composé d'une vessie excrétrice en V dont le sommet des branches est l'aboutissement d'un canal collecteur principal du type Mesostoma. Il a été dénombré avec certitude un total de $2 \times 8=16$ solénocytes: deux situés dans le deuxième coude des canaux cystogènes, deux au niveau de la paire antérieure des cellules granuleuses, deux au niveau de la paire postérieure et deux latéralement à la vessie. Il est vraisemblable que la formule excrétrice puisse être $2[(2+2)+(2+2)]=$ 16 ; elle serait alors la même que celle de la forme adulte; mais il nous a été toujours impossible de suivre le cheminement des canaux.

Ces cercaires assez actives, mobiles grâce à d'uniques mouvements de reptation, mais incapables de nager, ne sortent pas des sporocystes secondaires pour s'enkyster et se développer en métacercaires.

\section{$3^{\circ}$ Kystes des Métacercaires}

Dès que la cercaire a entrepris sa transformation, elle sécrète une enveloppe kystique transparente, très mince et déformable, qui croît en même temps qu'elle et s'épaissit progressivement en refoulant les parois du sporocyste qui se distendent. Les kystes des métacercaires mûres sont régulièrement sphériques, non déformés par la pression des kystes adjacents si nombreux soient-ils. Ils mesurent $135 \mu$ de $\emptyset$ environ. Leur paroi est épaisse de 11 à $12 \mu$ et extrêmement résistante, rendant le dékystement mécanique fort difficile et très aléatoire ; elle est formée de deux couches concentriques, intimement accolées sur tout leur pourtour, une interne de $6 \mu$ et une externe de 4-5 $\mu$. La métacercaire mûre, repliée sur elle-même deux fois, est peu mobile dans son kyste.

Ces derniers sont disséminés partout dans la région viscérale hépato-pancréatique et gonadique ; seul le pied musculeux échappe à l'envahissement. La survie des mollusques parasités par des kystes dont le nombre s'élève fréquemment à 200 ou 300 , pose une réelle énigme. Leur comportement est, apparemment du moins, peu perturbé par le parasitisme, quelque intense qu'il soit ; mais il n'a pas été établi si, comme chez les Peringia ulvae de Krull (1935) ou de Rothschild (1936, 1938, 1941), il y avait une répercussion sur la sexualité ou la croissance. Il est vraisemblable que les métacercaires mûres sont capables de survivre longuement à la disparition spontanée des sporocystes. On devrait sans doute pouvoir les retrouver au sein même de la masse glandulaire qui les héberge à certaines époques de l'année, les plus éloignées du moment de l'infestation miracidienne. 


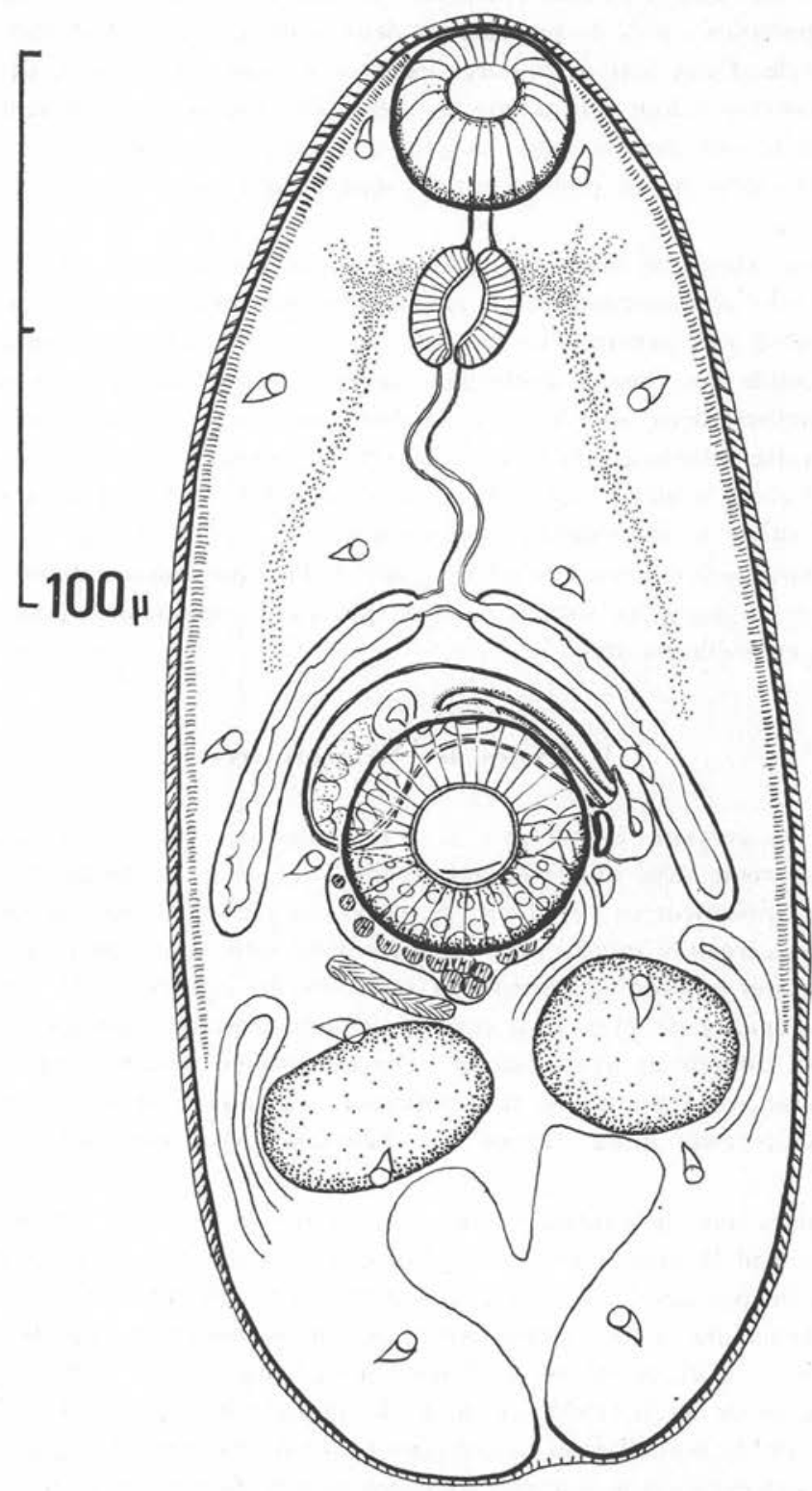

Fig. 4. - Maritrema syntomocyclus n. sp. - Métacercaire mûre dékystée. Vue ventrale. L'anneau des vitellogènes est toujours invisible. 


\section{Métacercaire}

La métacercaire artificiellement dékystée est relativement mal lisible. Son parenchyme est formé de grosses cellules qui masquent les contours des organes; elle apparaît de ce fait « inachevée », encore que parfaitement mûre. Elle est peu mobile. Sa description répond aux caractéristiques essentielles du genre Maritrema (fig. 4).

Forme du corps aplatie, plus longue que large, mesurant de $190 \times 90 \mu$ à $270 \times$ $120 \mu$. Un même exemplaire, suivant son degré d'élongation, mesurera à frais de $190 \times$ $100 \mu$ à $300 \times 70 \mu$.

La cuticule, épaisse de 2,5 à $3 \mu$, est totalement épineuse ; les épines en forme de pointe mesurent au niveau du pharynx $2,5 \times 1 \mu$ de large ; à partir de la ventouse ventrale, elles vont en s'atténuant vers la partie postérieure du corps mais sans disparaître complètement, même au niveau du pore excréteur. Ventouse orale sub-terminoventrale légèrement ovalaire de 28-32 × 20-27 $\mu$ de diamètre, ou parfois ronde de $31 \mu$ de diamètre. Ventouse ventrale de 35 à $40 \mu$ de diamètre, située au niveau de la mi-longueur du corps. Le bord des deux ventouses est glabre. Le rapport ventousaire V.O./V.V. $=1 / 1,4$ environ.

Prépharynx long de zéro à $25 \mu$. Pharynx ovoïde relativement volumineux de 17-20 $\times 14-16 \mu$. Esophage rectiligne long de 20 à $40 \mu$, parfois sinueux chez l'animal antérieurement contracté.

Les caeca mesurent de 60 à $85 \mu \times 7 \mu$; ils divergent juste en avant de la poche du cirre; leur fond atteint le niveau du bord postérieur de l'acétabulum sans le dépasser et n'est pas directement en rapport avec les testicules.

Appareil génital: Pore génital juxta-acétabulaire gauche.

Les testicules arrondis non lobés sont situés en arrière de l'ovaire, symétriquement par rapport à l'axe du corps. Taille $35 \mu$ de diamètre ou $34 \times 22 \mu$. Spermiductes non observés.

La poche du cirre est située à la périphérie de l'hémi-acétabulum antérieur, en arrière des caeca, symétriquement par rapport à l'axe du corps. Elle mesure $55 / 65 \times$ $15 / 17 \mu$. Sa paroi mince $(1 \mu)$ est nettement figurée sur toute sa périphérie. Elle contient une vésicule séminale toujours vide de spermatozoïdes, à laquelle fait suite un canal déférent long et contourné, assez épais, le long duquel ni la pars prostatica ni les cellules prostatiques ne sont aisément discernables.

Le canal déférent est susceptible de s'évaginer en un cirre glabre et épais long de 30-35 $\mu$ au moins $\times 8 \mu$ de diamètre. Il est vraisemblable que la vésicule séminale replète par la mise en activité des gonades mâles, puisse accroître les dimensions de la poche du cirre et lui faire occuper un emplacement légèrement différent chez le trématode adulte. Les spermiductes ne sont pas visibles. Le rapport de la longueur de la poche du cirre (à vésicule séminale vide) à la longueur du corps (P.C./L.C.) est égal à $1 / 4$.

L'ovaire est médian et sous-acétabulaire. Il mesure $35-40 \times 22 \mu$. Le départ de l'oviducte est situé au milieu de son bord postérieur. L'ootype cilié est parallèle à ce bord, légèrement décalé vers le côté droit. 
Le reste du carrefour ootypique n'est pas lisible, de même que la glande de Mehlis ; on ignore s'il existe un réceptacle séminal et un canal de Laurer.

Les contours des glandes vitellogènes ne sont pas apparents. Il nous paraît toutefois peu vraisemblable que les glandes vitellogènes puissent se situer ailleurs qu'en arrière des caeca; toute la zone qui leur est antérieure apparaît assez homogène et vide d'organe. Une anse utérine s'immisce entre le côté externe des testicules et la bordure correspondante du corps. Le métraterme n'est pas spécialement musculeux; il mesure 15 à $25 \mu$ environ. L'atrium génital est réduit, et ses parois sont minces.

Système excréteur: Sa topographie est celle classique des Microphallidés. On compte 16 solénocytes assez grands $(9-10 \mu)$, disposés selon la formule $2[(2+2)+$ $(2+2)]=16$. La vessie est en $\mathrm{V}$ en arrière des testicules.

\section{Discussion}

En dépit d'une insuffisance de description portant sur la non-mise en évidence de ia position réelle des vitellogènes, nous rapportons le distome, parmi les Microphallidés Ward, 1901, au genre Maritrema Nicoll, 1907 dont il présente sans exception toutes les caractéristiques; les risques d'erreurs nous paraissent à peu près nuls. L'utilisation de la clé diagnostique des espèces du genre Maritrema de Deblock et Combes, 1965 conduit (d'après la somme des caractères suivants : cirre cylindrique glabre ; poche du cirre à parois minces de longueur moyenne (P.C./L.C. $=1 / 4)$; ovaire médian sousacétabulaire ; canal éjaculateur long et contourné ; rapport ventousaire V.O./V.V. = $1 / 1,4$ ) aux reports successifs numéros $1,2,8$, et 10 . La clé restreint les investigations à trois Maritrema possibles: subdolum, acadiae et macracetabulum. Le rapport ventousaire des deux premiers est voisin de 1 , et leurs caeca sont très longs, dépassant assez largement le niveau du bord postérieur de la ventouse ventrale; subdolum évolue suivant une biologie toute classique (Deblock et coll., 1961) ; par contre, macracetabulum Deblock et coll., 1964, dont la biologie est inconnue, ne se différencie de la présente métacercaire que par sa taille, juste plus grande du double dans la totalité de ses dimensions et par un corps de forme plus orbiculaire. On ne peut donc pas éliminer tout à fait l'hypothèse que cette dernière espèce représente la forme adulte de nos métacercaires, une croissance chez l'hôte définitif n'étant pas à exclure a priori. L'infestation expérimentale de divers vertébrés, plusieurs fois répétée (canards, hamsters, souris), a malheureusement échoué dans tous les cas pour des motifs inconnus (spécificité de l'hôte définitif ?, altération du pouvoir évolutif des larves, consécutive à leur conservation prolongée au laboratoire ?...). Il n'est donc pas possible actuellement de rapporter avec certitude la métacercaire à une forme adulte déjà décrite. Nous la

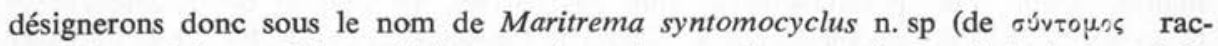
courci et xủxios cycle), en insistant néanmoins sur la parenté morphologique qu'elle présente avec Maritrema macracetabulum [espèce parasite de Nyroca ferina (L.) (Ansériforme) capturés en cours de migration dans la vallée de la Somme (France)], dont le corps est toutefois proportionnellement nettement plus large $(470 \times 300 \mu)$. 


\section{B) MICROPHALLUS SCOLECTROMA n. sp.}

HôTE DÉFINITIF : inconnu (oiseau malacophage ?) .

Localisation gÉOgRAPHiQUe: Golfe de Figari (Corse, France).

DATE DE RÉCOLTE : juillet 1965.

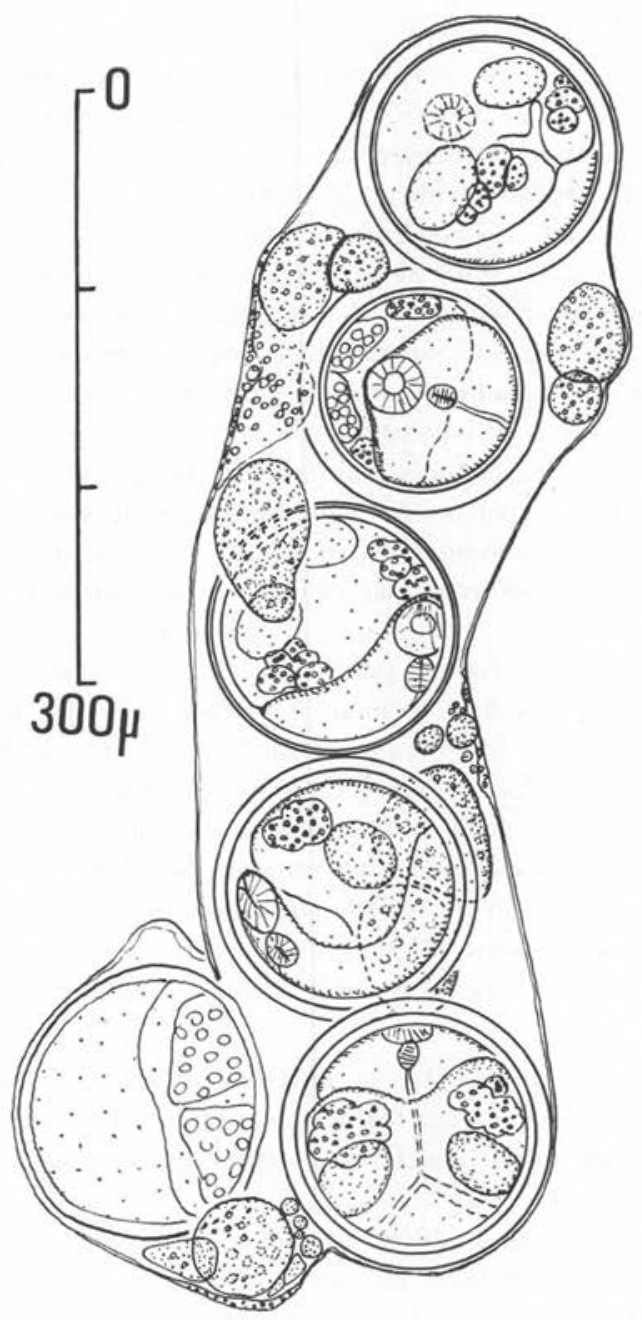

Fig. 5. - Microphallus scolectroma n. sp. Sporocyste isolé contenant cinq métacercaires mûres et une en voie de maturation. Bourgeons cercariens, cercaires et amas cellulaires indifférenciés. 
MATÉRIEL DE DESCRIPTION : Matériel vivant et fixé. Coupes histologiques.

Hôte INTERMÉdiaire: Hydrobia (= Paludestrina) acuta (Draparnaud). Mollusque Prosobranche Bithynellidé.

Sporocystes : Même aspect et mêmes caractéristiques que ceux de Maritrema syntomocyclus (voir fig. 5).

Cercaires : Le raccourcissement du cycle évolutif s'accompagne dans le cas de cette espèce d'une simplification morphologique encore plus poussée que dans le cas précédent ; la cercaire apparaît comme un stade passager fugace, simplement présent pour satisfaire les nécessités de l'ontogénèse des trématodes ; le parasite donne l'impression qu'il cherche à passer directement des bourgeons cercariens intra-sporocystiques à la métacercaire, en sautant le stade cercaire (fig. 6).

La taille varie suivant le degré de contraction ou l'élongation de la larve. A frais et à maturité, elle mesure 70-90 $\mu \times 55 \mu$ de large à l'état rétracté, et 100-150 $\mu \times$ 30-40 $\mu$ à l'état étiré. La cuticule est réduite à une membrane très mince d'un demimicron, non épineuse, mais finement annelée transversalement ; elle n'est garnie d'aucune soie cuticulaire sensitive. Elle constitue une sorte de sac empli de cellules arrondies au sein desquelles aucun organe ne se différencie, même dans les cercaires les plus mûres, à l'exception d'une ébauche de ventouse terminale qui ne se sépare du reste $\mathrm{du}$ corps que par une mince limitante peu visible; elle mesure 17-20 × 17-20 $\mu$ à l'état de repos mais peut se déformer par élongation pour atteindre jusqu'à $28 \times 12 \mu$ de large. Elle est dépourvue de stylet. Le corps cercarien ne présente ni ventouse ventrale ni glande cystogène. Le système excréteur se réduit à une vessie bilobée de 15 à $20 \mu$ de long, sans contours définis, donnant naissance à de très fins canaux primaires quasiment toujours invisibles. Un unique solénocyte n'a été décelé au tiers antérieur du corps quiune seule fois. La formule excrétrice demeure donc inconnue. Une telle cercaire inachevée, réduite à l'état d'ébauche à peine différenciée des bourgeons cercariens, est naturellement incapable de mouvements coordonnés de déplacement ou de nage dans l'eau ; elle est faiblement mobile sur place, destinée à passer rapidement au stade métacercaire, au sein même du sporocyste.

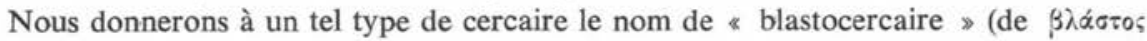
bourgeon).

Kystes des Métacercaires : Ils se développent de la même façon que dans l'espèce précédente. Une jeune métacercaire ne se différencie d'une blastocercaire mûre que par la présence d'une mince enveloppe kystique qui l'entoure et dans laquelle elle est à moitié repliée en deux.

Les kystes des métacercaires mûres, toujours inclus dans les sporocystes, envahissent la totalité de la masse viscérale du mollusque; ils sont régulièrement sphériques, non déformables par la pression des kystes adjacents, et mesurent 110 à $125 \mu$ de diamètre. Leur paroi est hyaline, épaisse de 6,5 à $8,5 \mu$ aux pôles et de 10-11 $\mu$ à l'équateur. Cette différence dépend d'une hétérogénéité de structure ; la membrane interne, épaisse de 5,5/6,5 $\mu$, est parfaitement sphérique ; la membrane externe épaisse de $2 \mu$ est très légèrement ellipsoïde car elle se décolle légèrement de la précédente selon 
un diamètre ; l'ensemble donne ainsi l'apparence d'une paroi à trois couches. Elle est très résistante et ne permet qu'un très difficile dékystement mécanique. La métacercaire dans son kyste se distingue de la précédente par la présence de masses granuleuses, constituées par les glandes vitellogènes assez aisément repérables au microscope au travers de la paroi du kyste.

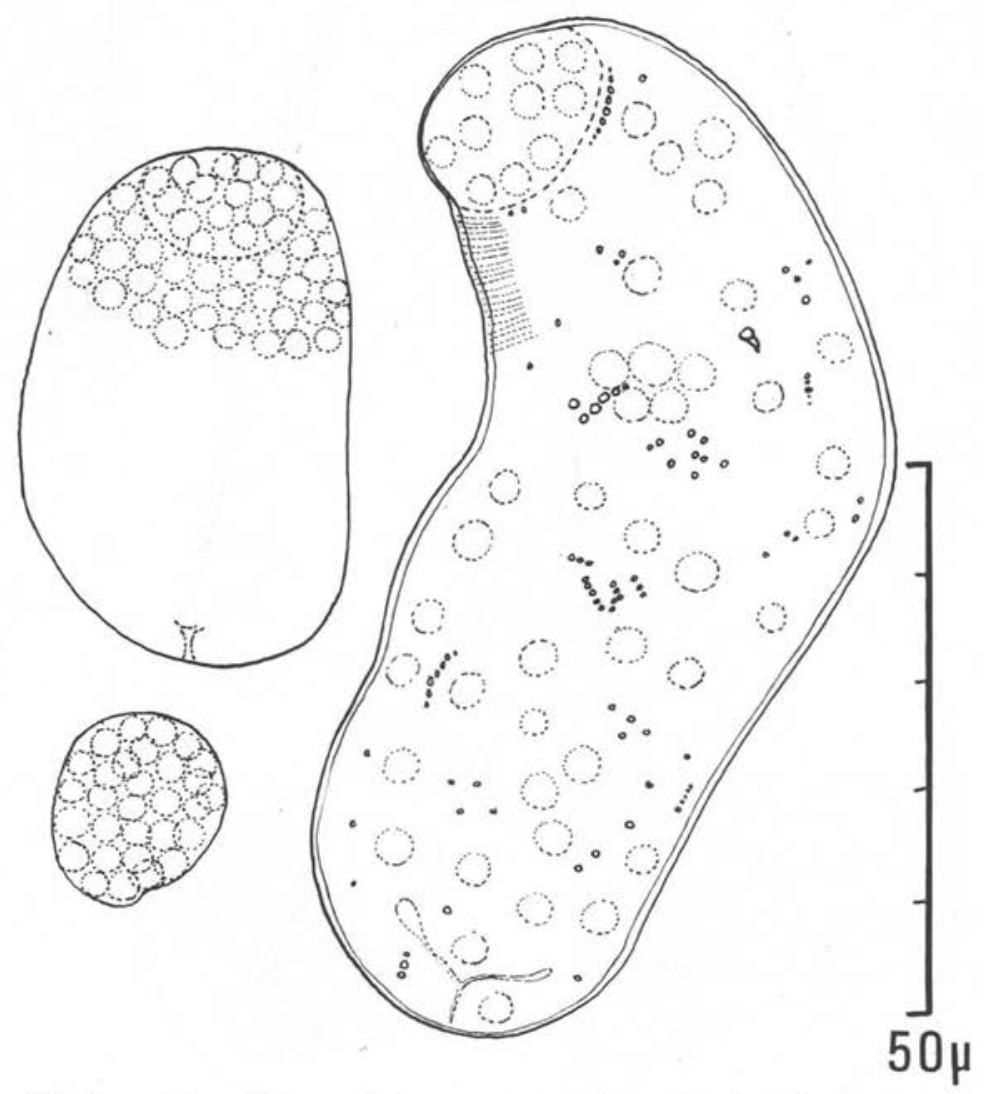

Fig. 6. - Microphallus scolectroma $\mathrm{n}$. sp. - A gauche, deux bourgeons cercariens immaturés; à droite, blastocercaire mûre, vue ventrale; la striation cuticulaire n'a été figurée partiellement qu'en dessous de la ventouse orale

Métacercaires : La métacercaire mûre artificiellement dékystée paraît complètement achevée; elle est assez mobile dans son kyste. Sa description répond à toutes les caractéristiques du genre Microphallus (fig. 7).

Corps lancéolé aplati dorso-ventralement; extrémité antérieure allant en s'amincissant et extrémité postérieure arrondie.

Taille: 300 à $340 \mu \times 170-190 \mu$ de largeur maximale au niveau de la ventouse ventrale. 


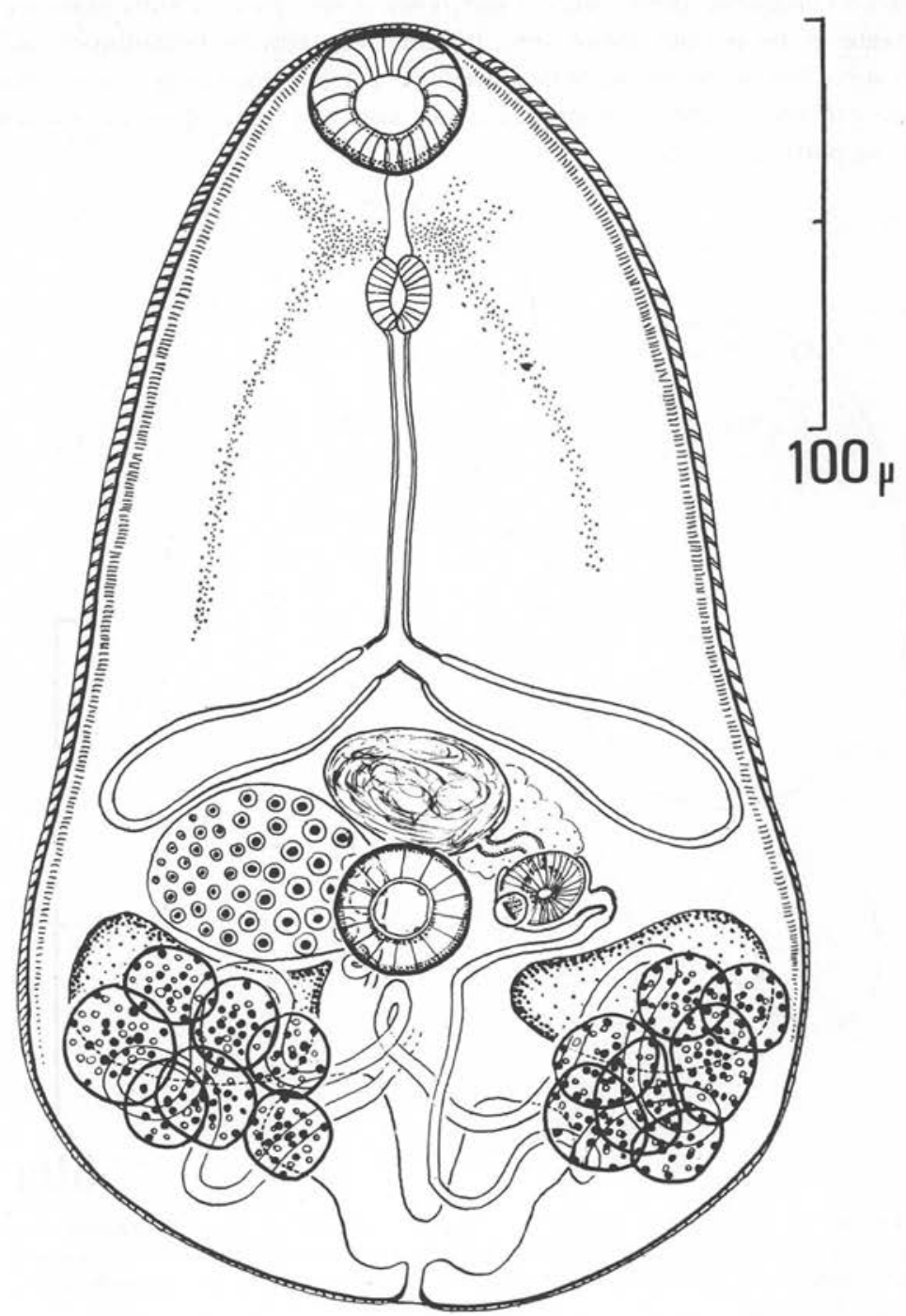

Fig. 7. - Microphallus scolectroma n. sp. - Métacercaire mûre dékystée. Vue ventrale.

La cuticule, épaisse de $2 \mu$, porte à la partie antérieure des épines fines mesurant à hauteur du pharynx $2 \times 1 \times 1 \mu$ et allant en s'atténuant jusqu'au niveau des fonds des caeca pour n'être plus représentées au-delà que par une fine ponctuation. 
Ventouse orale subtermino-ventrale, légèrement ovalaire, de 36 à $40 \mu$ de diamètre. Ventouse ventrale circulaire de 32 à $34 \mu$ de diamètre située au niveau des 4/6 de la longueur du corps (l'utérus étant vide d'œufs).

Prépharynx long de zéro à $25 \mu$. Pharynx ovoïde de $20 \times 15$ à $22 \times 17 \mu$. Esophage rectiligne, long de 60 à $95 \mu$ en fonction de l'état d'extension de la partie antérieure du corps, sur $4 \mu$ de diamètre. Caeca divergents relativement courts, de 70-95 $\times$ 17-20 $\mu$ de diamètre ; leur fond ne dépasse pas le bord postérieur de l'acétabulum.

Appareil génital: Les testicules ovalaires, à grand axe transversal, se situent en arrière de l'ovaire, symétriquement par rapport à l'axe du corps. Taille: $30-40 \times$ 55-65 $\mu$. Spermiductes issus de leur bord antéro-interne, convergeant sous la ventouse ventrale en un spermiducte commun qui aborde la partie postéro-médiane de la vésicule séminale. Absence de poche du cirre. Vésicule séminale ovoïde située juste en dessous de la bifurcation œsophago-caecale, mesurant suivant sa réplétion par les spermatozoïdes, de $36 \mu$ de diamètre à $36 \times 50 \mu$. Le canal déférent, long de 20 à $25 \mu$, ne décrit pas de boucle en cheminant dans l'axe de la glande prostatique. Pars prostatica située à la base de la papille mâle. Papille mâle charnue de forme sphéroïdale, prenant un aspect en tronc de cône par ap'atissement à frais, évoquant celui de $M$. claviformis; elle mesure $17 / 19 \mu$ de diamètre $\times 17 / 18 \mu$ de hauteur. En vue apicale, elle apparaît comme un simp'e anneau charnu assez régulier, plus petit que la ventouse ventrale (fig. 8).

L'ovaire est situé à droite de l'acétabulum et à son niveau, entre le caecum et le testicule droit. Il est de forme générale ovalaire et mesure $45 \times 55 / 60 \mu$. L'anatomie du carrefour ootypique n'a pas été précisée. L'utérus ne dépasse pas antérieurement la limite constituée par le fond des caeca; vide d'œufs, il décrit trois larges boucles du côté droit, d'arrière en avant, passe du côté gauche pour suivre un trajet symétrique mais de sens inverse et monte directement jusqu'à l'atrium génital qu'il aborde par le côté gauche sans présenter de différenciation métratermique très accusée. La progénèse uniquement protandrique de la métacercaire n'a pas permis l'observation des œufs.

Les vitellogènes sont groupés en amas de follicules granuleux nettement figurés situés en arrière des testicules et ventralement. Les vitelloductes convergent en direction de la région post-acétabulaire et forment un petit réservoir vitellin médian.

Le système excréteur est formé d'une vessie en $\mathrm{V}$ à branches courtes ne dépassant pas la mi-distance testicules-extrémité postérieure du corps. La formule excrétrice est du type classique $2[(2+2)+(2+2)]=16$.

\section{Discussion}

Parmi la trentaine de représentants du genre Microphallus actuellement décrits, considérer la combinaison de caractères morphologiques suivante: taille du corps $(300-350 \mu)$, taille et conformation de la papille mâle (en forme de sphère ou de tronc de cône de $18 \times 18 \mu$ de $\emptyset$ ) conduit à éliminer : $1^{\circ}$ les espèces de taille corporelle ana- 
logue qui présentent une papille beaucoup trop petite (limuli Stunkard, 1951:6 $66 \mu$ ) ou beaucoup trop grande (bilobatus Cable et coll., 1960:35 $\times 45 \mu$ ); $2^{\circ}$ les espèces de taille inférieure à papille très réduite (somateriae (Kulatschkova, 1958) et fusiformis Reimer, $1963: 7-8 \mu(5) ; 3^{\circ}$ les espèces de taille corporelle immédiatement supérieure qui possèdent des papilles de conformation différente ; en pain de sucre asymétrique de $25 \times 30 \mu$ (pygmoeum Levinsen, 1881); en cylindre fin et allongé de $24 \times 6 \mu$ (gracilis Baer, 1943); en cylindre plus épais de $20 \times 15 \mu$, à fin méat subterminolatéral (enhydrae Rausch et coll., 1951) ; ou de forme complexe et volumineuse de $55 \times$ $55 \mu$ [turgidus (Leigh, 1958)]. Les espèces de ce premier lot nous paraissent ainsi toutes
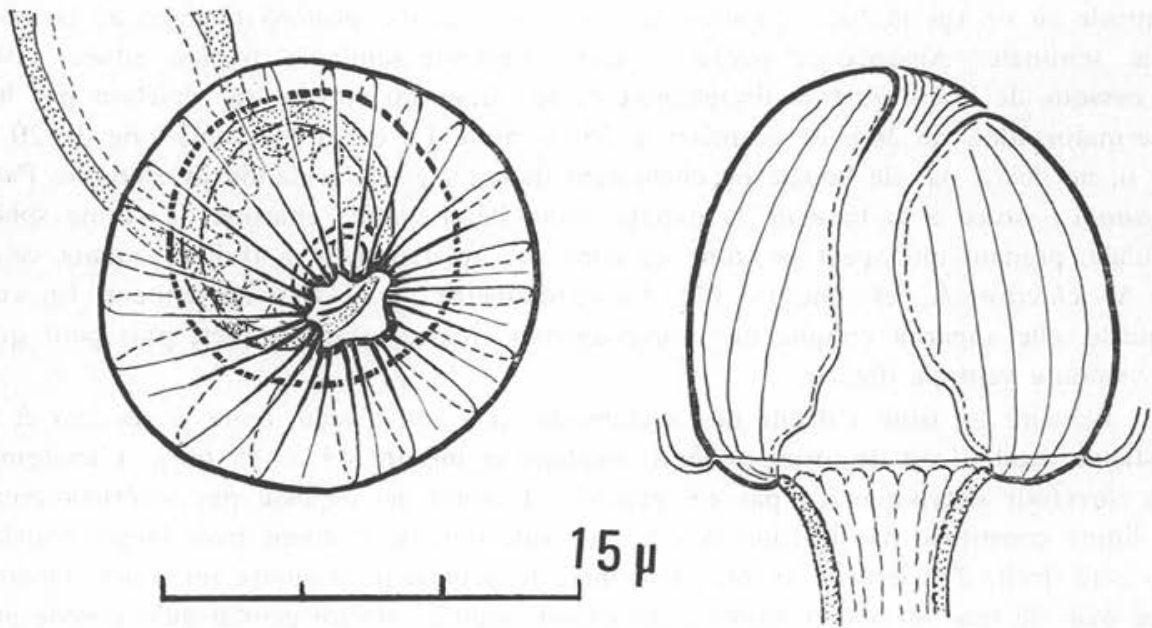

FIG. 8. - Microphallus scolectroma n. sp. - Papille mâle. A gauche vue apicale. A droite, élévation

différentes du Microphallus de Corse. A ces raisons morphologiques s'ajoutent d'ailleurs aussi des arguments d'ordre écologique et géographique; plusieurs des Microphallus mentionnés ci-dessus n'ont été décrits que dans le Nouveau Monde (limuli, bilobatus, turgidus); ou dans le Pacifique Nord (enhydrae); certains évoluent dans le milieu marin où ils trouvent mollusques (pygmoeum) et seconds hôtes intermédiaires (limuli, enhydrae) ; d'autres évoluent très probablement chez des mollusques d'eau douce (gracilis, turgidus).

Ces éliminations laissent encore subsister un groupe de quatre trématodes de morphologie fort homogène dont les tailles du corps s'échelonnent d'une façon continue de 250 à $500 \mu$, et celles des papilles de $9 / 10$ à $19 / 21 \mu$; la forme de ces derniers organes est circulaire et dépourvue de la moindre singularité. Il se pourrait que la métacercaire de Corse corresponde à l'une d'entre elles car nous ignorons de

(5) Nous considérons cette dernière espèce morphologiquement plus voisine des Gymnophallidés que des Microphallidés. 
combien sa taille est capable de croître chez l'hôte définitif. Il s'agit de $M$. calidris Belop. et coll. 1963, capellae (Yamag., 1939), claviformis (Brandes, 1888) et oedemia (Belop., 1952). Néanmoins, une donnée complémentaire récente permet en toute certitude de discriminer $M$. claviformis, la plus petite des espèces de ce lot $(220-300 \mu)$. Sa biologie vient en effet d'être décrite (in Deblock et coll., 1965): la cercaire est une xiphidio-cercaire monostome agastre leptocerque à 12 protonéphridies et à cuticule garnie de sensilles ; elle s'enkyste généralement chez des crustacés Isopodes ou Amphipodes; elle ne présente par conséquent aucun point commun tant morphologique que biologique avec la blastocercaire de l'espèce décrite: à l'analogie des formes métacercaires et adultes correspond une disparité accusée des formes larvaires qui exclut l'hypothèse que $M$. claviformis soit susceptible d'évoluer selon deux modalités différentes - l'une à cycle long et l'autre à cycle court - en fonction d'une différence de climat par exemple.

D'après les données de la littérature actuelle, nous ne sommes pas capables de discriminer avec certitude les autres espèces du dernier groupe en discussion (sauf peut-être capellae Yamag. dont la papille est plus nettement volumineuse $-20 / 33 \mu$ pour une taille de 400 à $500 \mu$ ). Aussi préférons-nous attendre de nouvelles précisions morphologiques ou biologiques concernant ces espèces avant de rapporter le distome méditerranéen à l'une ou l'autre d'entre elles ; nous lui conférerons ainsi provisoirement le statut d'espèce nouvelle, sous le nom de Microphallus scolectroma n. sp., évocateur des particularités évolutives de la larve (de $\sigma \times \omega \dot{\lambda} \eta \eta 弓$

Il est possible que soient identifiables à $M$. scolectroma, où à une espèce voisine évoluant comme elle :

$1^{\circ}$ Metacercaire pirum Lebour, 1907 (kyste de $80 \mu$ et larve de $200 \mu$ à ventouse de $20 \mu$ ), trouvée dans l'hépatopancréas de Paludestrina stagnalis en Grande-Bretagne ; $2^{\circ}$ Microphallus claviformis sensu Reimer, 1963, dont les kystes ont été trouvés dans l'hépato-pancréas du même hôte en mer Baltique ; $3^{\circ}$ un adulte mûr, trouvé à l'autopsie d'un Hoematopus de Vaderroärn (Suède) en juillet 1906, faisant partie des collections de Jaegerskioeld, mais non identifié par cet auteur.

\section{4. - CONCLUSIONS}

Maritrema syntomocyclus s'inscrit dans le sous-groupe A, à côté de Maritrema oocysta et de Microphallus somateriae; Microphallus scolectroma, dans le sousgroupe $\mathrm{B}$, section 1 , à côté d'Atriophallophorus minutus.

Les modalités du cycle évolutif de ces deux Microphallidés confirment que les représentants de la famille manifestent une tendance remarquable à l'abrègement de leurs cycles. Ils réalisent cet abrègement de multiples façons : $1^{\circ}$ par suppression du stade miracidial libre ; $2^{\circ}$ par suppression des stades cercaires libres et suppression concomitante du second hôte intermédiaire; il s'ensuit une maturation des formes infestantes chez le mollusque, premier hôte intermédiaire ; cette modalité évolutive entraîne une tendance, $3^{\circ}$ à la suppression du stade cercaire lui-même (remplacé par 
la blastocercaire), et $4^{\circ}$ à la suppression de l'enkystement de la métacercaire ; enfin, $5^{\circ}$ la plupart des espèces montrent un état de progénèse protandrique de leur métacercaire accompagné d'un état extrêmement voisin de la progénèse gynandrique.

On peut concevoir hypothétiquement la filiation phylétique du phénomène. Il est vraisemblable qu'il tire son origine dans l'acquisition par la cercaire de la faculté de poursuivre son évolution dans les tissus du mollusque; dès lors, elle ne quittera plus le sporocyste. Le grand retentissement de cette faculté nouvelle est la suppression du second hôte intermédiaire Crustacé. Dans un premier temps, les sporocystes continuent néanmoins à produire des cercaires de morphologie classique, encore adaptées à une vie libre qu'elles ne mèneront pas; queue natatoire, stylet, glandes nécessaires à la pénétration au travers de la cuticu'e du second hôte, glandes sécrétrices du kyste de protection assurant la longue survie, devenus inutiles, ne tardent pas à disparaître secondairement, d'une façon progressive: queue et stylet d'abord, puis glandes de pénétration. Enfin, le terme ultime de l'évolution tend à réaliser la suppression du stade cercaire lui-même, auquel se substitue la blastocercaire ; le parasite ne conserve de la chaîne des stades classiques que le sporocyste, générateur des bourgeons rapidement transformés en métacercaires infectieuses, lesquelles n'éprouvent pas toujours la nécessité de s'enkyster; leur état de progénèse est destiné à écourter la période de maturation génitale chez l'hôte définitif; il représente le point final des processus d'accélération du cycle biologique des parasites.

Les avantages d'une telle solution se conçoivent ; elle évite le gaspillage des cercaires, entraînés par l'aventure du stade libre, au cours duquel le fragile et éphémère organisme doit s'installer chez le second hôte intermédiaire qui aura sollicité ses tropismes, s'il en trouve un. Evoluer chez le mollusque assure au parasite un avenir moins incertain ; il restreint d'ailleurs sa production, car tout bourgeon est assuré de sa transformation en métacercaire. L'accumulation progressive de ces formes chez le mollusque infesté est un facteur qui favorise des infestations rares peut-être, mais souvent massives (une, deux ou trois centaines de vers par prise). Cependant ces solutions originales présentent aussi des inconvénients: le trématode risque de diminuer ses chances de parvenir au stade adulte : $1^{\circ}$ il restreint l'aire de dispersion de ses formes infestantes par une diminution de leur mobilité ; $2^{\circ}$ il restreint la variété de ses présentations capables de tenter l'appétit de l'hôte vertébré ; $3^{\circ}$ il restreint l'éventail des vertébrés susceptibles de les ingérer, en les limitant aux seuls animaux volontiers malacophages.

Il est possible que dans la nature, les avantages compensent les inconvénients. D'après les données de la littérature, il ne semble pas que les espèces ayant acquis une telle biologie soient plus florissantes, par leur abondance et leur fréquence, que les espèces à biologie classique, au contraire. Mais ce n'est peut-être là qu'une apparence, qui peut d'ailleurs tenir à d'autres causes dont nous n'avons pas notion ; il est concevable en effet que ces espèces ne maintiennent justement leur existence que grâce à l'abrègement de leur cycle. 


\section{RÉSUMÉ}

Les auteurs décrivent Maritrema syntomocyclus n. sp. et Microphallus scolectroma n. sp. parasites de Hydrobia (= Paludestrina) acuta (Drap.), mollusque Prosobranche d'un gîte des côtes méditerranéennes (Corse). Ces espèces accomplissent la totalité de leur cycle évolutif larvaire chez le même mollusque. Il n'y a donc pas de second hôte intermédiaire. Les hôtes définitifs sont inconnus. La cercaire du Maritrema est une Xiphidiocercaire monostome anentérique, secondairement inerme et anoure, à deux paires de glandes, et de formule excrétrice du type $2[(2+2)+(2+2)]=16$. La cercaire du Microphallus est une blastocercaire (cercaire ébauchée), dépourvue d'organes. L'une et l'autre s'enkystent dans les sporocystes qui les ont engendrées. La métacercaire de $M$. syntomocyclus rappelle morphologiquement $M$. macracetabulum Deblock et coll., 1964, et celle de $M$. scolectroma, M. calidris Belop. et coll., 1963, ou oedemia (Belop., 1952).

\section{Bibliographie}

Afanassief (V. P.), 1941. - In Belopolskaïa (M. M.), 1952, p. 711-712, fig. 204.

BAeR (J. G.), 1943. - Les trématodes parasites de la musaraigne d'eau Neomys fodiens (Schreb.). Bull. Soc. Neuchâtel Sci. Nat., 68, p. 33-84.

Belopolskaïa (M. M.), 1949. - Cycle évolutif de Spelotrema pygmoeum parasite d'oiseau. Dokl. Acad. Nauk SSSR, nov. ser., 66, n 1, p. 133-135.

Belopolskaïa (M. M.), 1952 et 1963. - La famille des Microphallidae, Travassos, 1920, in : Trématodes des animaux et de l'homme, de K. I. Skriabine. a) 1952, 6, p. 619-756; b) 1963,21 , p. $259-504$.

Belopolskaïa (M. M.), 1962. - Les cycles évolutifs chez les trématodes de la famille des Microphallidae Travassos, 1920. Vestn. Leningr. Universit.. Ser. Biol., 3 (1), p. 45-53.

Brandes (G.), 1888. - Helminthologisches. Arch. f. Naturg. 54, 247-251.

BURNS (W. C.), 1963. - The occurrence of Levinseniella minuta (Trematoda : Microphallidae) in Oregon. Jl Parasitol., 49, p. 856.

Cable (R.), Connor (R.) et Balling (J.), 1960. - Digenetic trematodes of Puerto-Rican shore birds. Sc. Survey of Porto-Rico and the Virgin Islands, 17, part 2, p. 187255, 48 fig.

Deblock (S.) et Combes (C.), 1965. - Contribution à l'étude des Microphallidae Travassos, 1920 (Trematoda). X. Maritrema pyrenaica n. sp., parasite du desman (mammifère insectivore). Essai de clé diagnostique des espèces du genre. Bull. Soc. Zool. France, 90 , p. 101-117, 3 fig.

Deblock (S.) et Rosé (F.), 1964. - Contribution à l'étude des Microphallidae Travassos, 1920 (Trematoda) des oiseaux de France. IX. Description d'Anacetabulitrema samarae n. gen., n. sp., de Maritrema macracetabulum n. sp. et de Microphallus somateriae (Kulatsch.) n. comb., parasites d'Anatidés. Bull. Soc. Zool. France, 89, p. $429-443$. 
Deblock (S.) et Rosé (F.), 1965. - Contribution à l'étude des Microphallidae Travassos, 1920 (Trematoda) des oiseaux de France. XI. Identification de la cercaire de Microphallus claviformis (Brandes, 1888). Bull. Soc. Zool. France, 90, p. 299-314.

Deblock (S.), CAPron (A.) et Rosé (F.), 1961. - Contribution à l'étude des Microphallidae Travassos, 1920 (Trematoda). IV. Le genre Maritrema Nicoll, 1907 ; cycle évolutif de $M$. subdolum Jaegerskioeld, 1909. Parasitologia, 3 (1-2), p. 105-119, 3 fig.

KrULL (M.), 1935. - Anatomische Untersuchungen au einheimischen Prosobranchieren und Beiträge zur Phylogenie der Gastropoden. Zool. Jb. Anat., 60, 399-464.

Kulatschkowa (V. G.), 1958. - Revue écologique et faunistique de la faune parasite des eiders communs des golfes du Kandalakchska. Travaux de la Réserve d'Etat du Kandalakchska 1958,1 , p. 103-159.

Lebour (M. V.), 1907. - Larval trematodes of the Northumberland coast. Trans. Nat. Hist. Soc. Northumb., N. Ser., 1, p. 437-454 et 500-501.

LeIGH (W. H.), 1958. - Carneophallus turgidus sp. nov. (Trematoda Microphallidae) from the raccoon (Procyon lotor) in South Florida. Jl. Parasit., 44 (1), p. 100-102.

Levinsen (G. M. R.), 1881. - Bidrag til kundskab om Groenlands Trematod fauna. Overs. Danske Vidensk. Selsk. Forkdl. $\mathrm{N}^{\circ} 1,1881$. Kjøbenhaun.

MARKowsKi (S.), 1936. - Ueber die Trematoden fauna der baltischen Mollusken aus der Umgebung der Hallinsel Hel. Bull. Akad. Polon. Sc. et Lettres, ser. B., Sc. Nat., II, p. 285-317, pl. 13, 14 et 15 .

RebeCQ (J.), 1960. - Présence en France de Maritrema subdolum Jaegerskioeld, $1909=$ Maritrema rhodanicum Carrère, 1936 (Trematoda Microphallidae). Vie et Milieu, 11 (1), p. 69-74.

REBECQ (J.), 1964. - Recherches systématiques, biologiques et écologiques sur les formes larvaires de quelques trématodes de Camargue. Thèse Sciences, Aix-Marseille, 223 p., 15 planches.

ReImer (L.), 1963. - Zur Veibreitung der Adulti und Larvenstadien der Familie Microphallidae Viana, 1924 (Trematoda, Digenea) in der Mittleren Ostsee. Zeitsch $f$. Parasitenk., 23, p. 252-273, 12 fig.

Rothschild (M.), 1936. - Gigantism and variation in Peringia Ulvae Pennant, 1777 caused by infection with larval trematodes. Jl Mar. Biol. Ass. U.K. 20, p. 537-546.

RothschILD (M.), 1937. - Note on the excretory of the trematode genus Maritrema Nicoll, 1907, and the systematic position of the Microphallinae Ward, 1901. Ann. Mag. Nat. Hist., ser. 10,19 , p. 355-365.

RoTHSCHILD (M.), 1938. - Further observations on the effect of trematode parasites on Peringia ulvae (Pennant 1777). Novit. Zool., 41, p. 84-102.

Rothschild (M.), 1941. - Observations on the growth and trematode infections of Peringia ulvae (Pennant, 1777) in a Jool in the Tamar Saltings, Plymouth. Parasitol., 33, p. $406-415$.

(Travail du Laboratoire de Parasitologie de la Faculté mixte de Médecine et Pharmacie de Lille. Directeur: Pr J. Biguet) 


\section{ADDENDUM}

Nous devons à la courtoisie de M. J. Rebecq, auquel nous renouvelons nos plus vifs remerciements, d'avoir pu consulter depuis le dépôt de notre manuscrit plusieurs préparations de deux Microphallidés obtenus expérimentalement chez un caneton ayant ingéré des métacercaires d'Hydrobies de Camargue. $1^{\circ} \mathrm{La}$ première, correspondant Levinseniella $s p$. Rebecq, 1964, se confond avec Microphallus somateriae (Kulatsch.) Deblock et Rosé, 1964. $2^{\circ}$, La seconde, un Maritrema, est pratiquement indiscernable de l'espèce décrite dans l'article ci-dessus, bien que les caeca soient un peu plus longs, dépassant le niveau du bord postérieur de l'acetabulum; les vitellogènes y forment un anneau complet en position classique. $\mathrm{Si}$ cette identité se confirmait ultérieurement, nous aurions acquis la preuve que $M$. syntomocyclus et $M$. macracetabulum constituent effectivement deux espèces distinctes. 